\title{
The Ups and Downs of Paste Transport
}

\author{
L. Pullum Australia
}

L.J.W. Graham, M. Rudman, B. Aldham, R. Hamilton CSIRO Manufacturing and Infrastructure Technology, Melbourne, Australia

\section{INTRODUCTION}

Mineral tailings pipelines have to traverse undulating terrain. Paste and high concentration tailings lines convey non-Newtonian slurries and usually contain coarse particles, i.e. $>20 \mu \mathrm{m}$, that are conveyed as a burden. In presentations at previous Paste conferences papers have been presented that demonstrated that such flows, while appearing to behave homogenously in fact stratify and require higher transport pressure gradients and more care when conveying than their true homogenous paste counterparts, (Pullum and Graham 2000, Pullum 2003, Talmon and Mastbergen 2004). The flows are most readily described using a stratified model and a non-Newtonian version of this type of model has been shown to predict such flows quite well (Pullum et al., 2004). While suitable non-Newtonian models have been devised for transport in horizontal lines the effect of incline on such hybrid suspension flows is yet to be established. A new tilting pipeline rig has been constructed at CSIRO to investigate the behaviour of these complex suspensions and this paper describes this new test facility and reports on preliminary results obtained with a visco-plastic suspension, typical of many non-Newtonian co-disposal systems, e.g. (Houman 2003).

\section{INCLINED PIPELINE TEST FACILITY}

A schematic of the test facility is shown in Figure 1. The loop has been specifically designed for high concentration suspension flows. The agitated mixing tank is fully instrumented and features a dual impeller set with one high solidity fluid foil impeller near the bottom of the tank and one lower solidity impeller near the top in accord with the experience of the authors with these types of flows e.g Graham et al., 2005. A two stage centrifugal pump set is used. The first stage is a 4 × 3 Warman 3 AHF, the head characteristics of which have been shown to be almost insensitive to yield stresses up to $200 \mathrm{~Pa}$ or high apparent viscosities, e.g. $\mathrm{Xu}$ et al., 2002. The second stage, is a conventional 4 × 3 GIW LCC-M80-300. Both pumps are equipped with mechanical seals to prevent dilution of the suspensions under test with gland water. The inclinable pipes are made from clear PVC allowing observation of the suspensions along the entire loop, and bends are restricted to normal long radius bends $(\mathrm{R} / \mathrm{D}=1.5)$ as the typically high viscosity of the suspensions prevents dramatic reorganization of the flow at the bend and reestablishment lengths are therefore quite small. Pressure gradients are monitored using several differential pressure transducers along the second half of each loop leg, enabling acceleration pressures, indicative of flow establishment to be measured if they are present. 


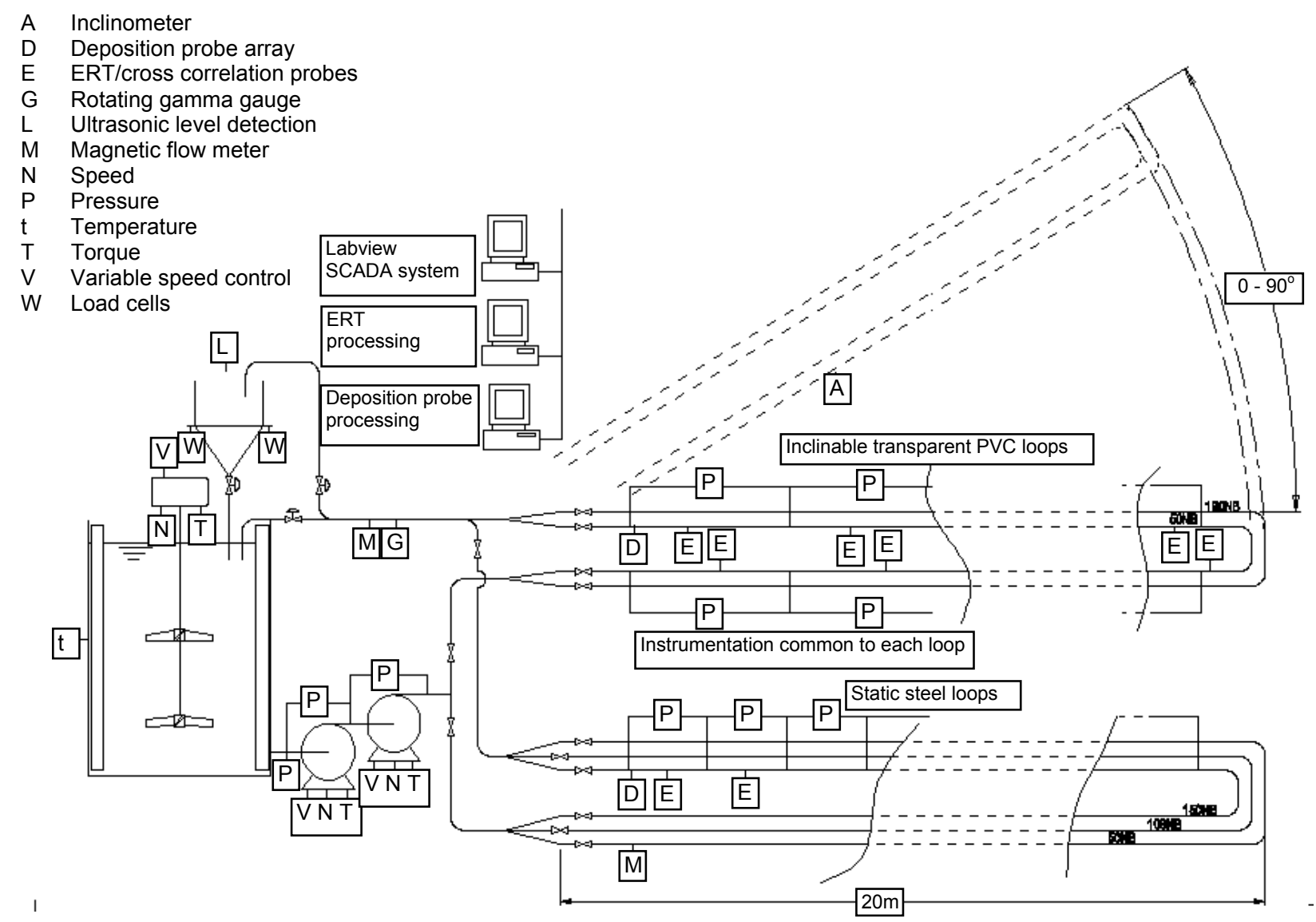

Figure 1 Schematic of the inclinable pipe test facility

Bulk flow rates are measured using either conventional magnetic flow meters or by diversion into an integral weigh tank, where the volumetric flow and mass flow rates are measured as a function of time. In-line density is measured with a gamma gauge that rotates around a vertical pipe section, ensuring that any stratification within the flow does not alter the measurement of the total in-situ concentration. This measurement is used to calibrate the tomography spools.

Electrical resistance tomography spools (ERT) are distributed around both legs of the loop and in the vertical section containing the gamma gauge. These spools are installed either singly or in pairs so that crosscorrelation methods may be used to establish the concentration and velocity distributions within the pipe. Two ERT systems are used, an ITS P2000 and a UCT development system, the latter being capable of scan rates up to $1000 \mathrm{~Hz}$.

Pairs of deposition probes (Erocolani et al., 1979) each separated by a small axial distance are fitted in pairs around the pipe and will be used to measure particle velocities close to the wall around the pipe's circumference.

The lines can be inclined at any angle from horizontal to vertical with suitable choking being provided in the return leg to prevent slack flow from occurring. 
The rig is controlled by using a PID system incorporated into the Labview SCADA system, and can be preset to any velocity (or Reynolds number) over the entire range of operation $0-4 \mathrm{~m} \mathrm{~s}^{-1}$ in the $100 \mathrm{NB}$ lines.

The inclined section is shown in Figure 2.

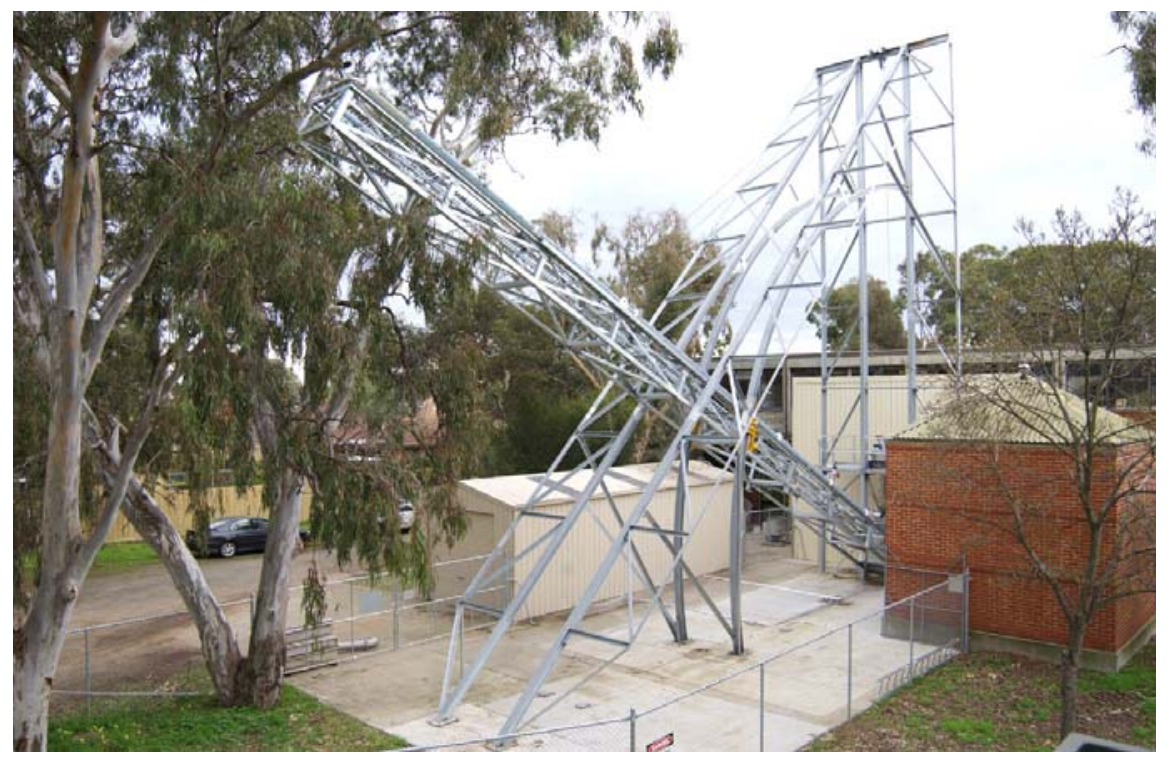

\section{Figure $2 \quad 20 \mathrm{~m}$ long fully inclinable pipe test loop}

\section{EXPERIMENTAL RESULTS}

\subsection{Suspension properties}

A model suspension to simulate a co-disposal system was used in these preliminary studies, whereby the non-Newtonian nature of the suspension attributable to the fine and slimes fraction in a real system was modelled using a clear polymer gel, Ultrez 10, which approximates a yield pseudoplastic typical of such slurries. The coarse fraction of the co-disposal system was modelled using crushed glass. This material has the benefits of having a similar immersed density to waste material, a similar angular shape and is rheologically "inert", i.e. it does not modify the underlying rheological character of the conveying slurry or carrier fluid. A typical steady shear rheogram for the carrier fluid is shown in Figure 3. 


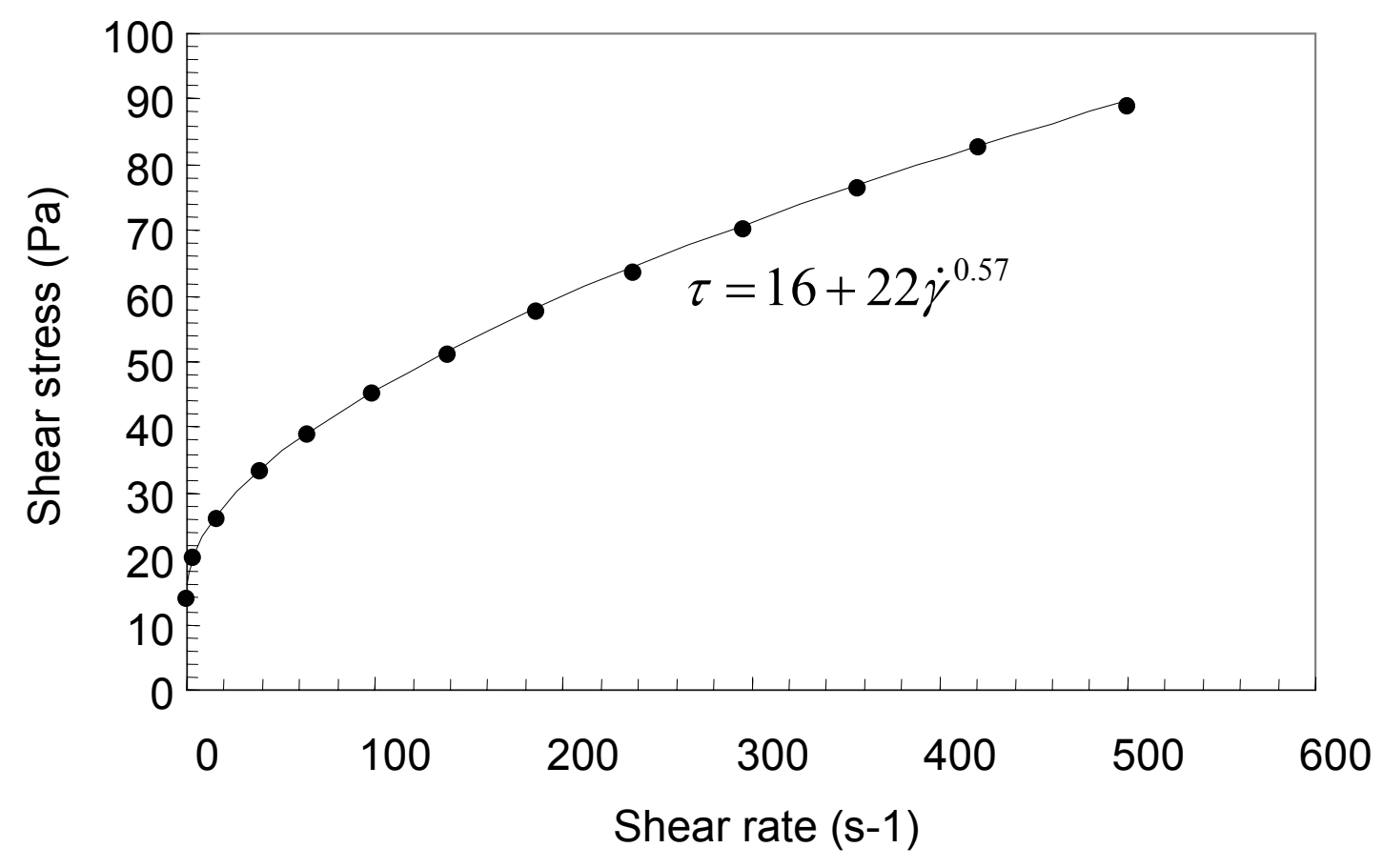

Figure 3 Steady shear rheogram for a $0.12 \% \mathrm{w} / \mathrm{w}$ aqueous suspension of Ultrez 10

It should be noted that at these concentrations Ultrez 10 does not exhibit any measurable elastic effects. The particle size distribution for the glass coarse burden is shown in Figure 4.

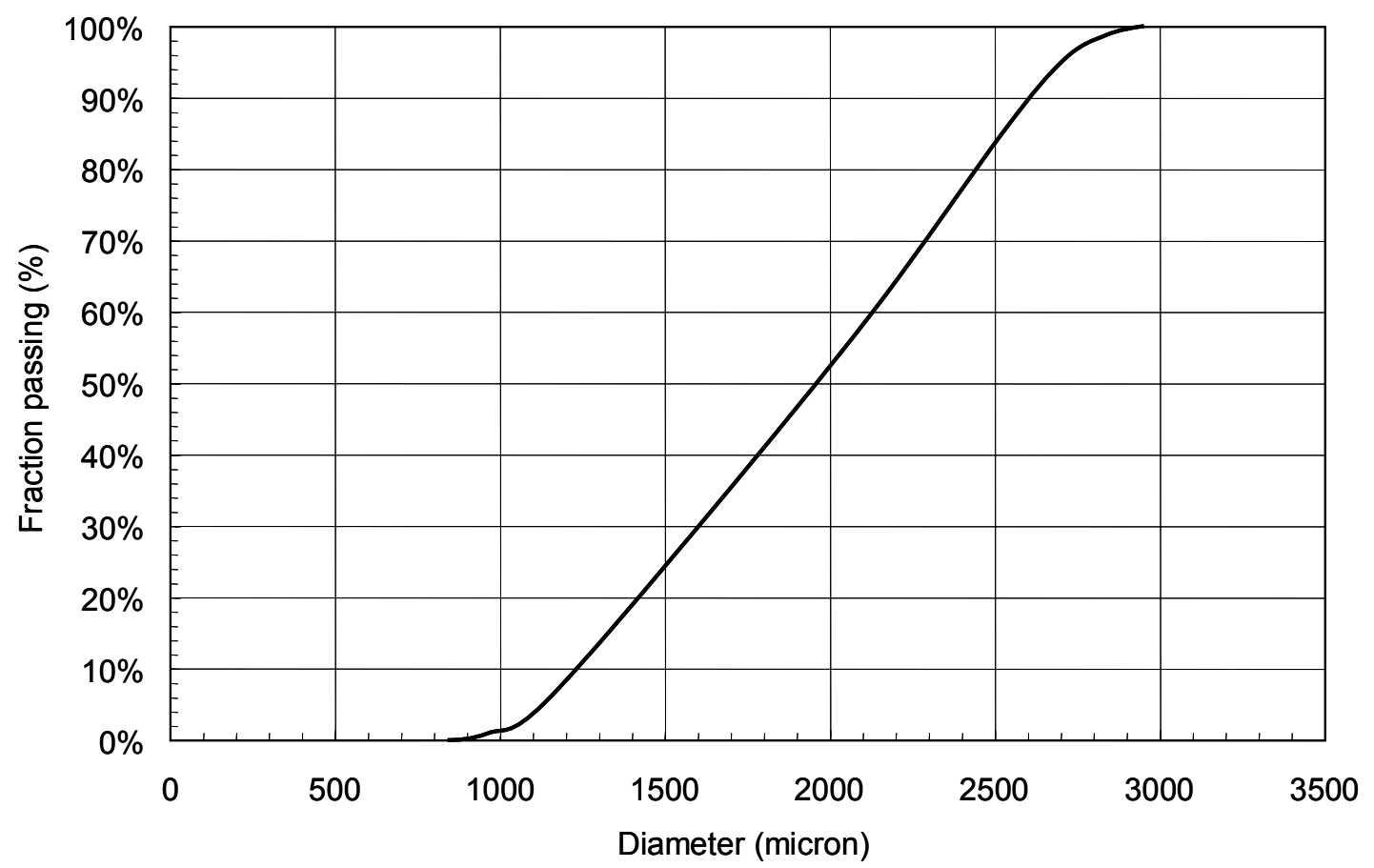

Figure 4 Particle size distribution of crushed glass coarse burden 
The minimum yield stress required for static stability is given by:

$$
\tau_{y} \geq \operatorname{kgd}\left(\rho_{s}-\rho_{f}\right)
$$

where $\mathrm{k} \sim 0.1$ for typical mineral particles. Substituting the material properties into this equation and using the particle top size, i.e. $3 \mathrm{~mm}$, gives a minimum required yield stress of $4.7 \mathrm{~Pa}$. This is less than a third of the yield stress of the carrier fluid from which we can conclude that the particles would be stable, i.e. would not settle under static conditions. In other words they would appear to form a homogeneous, albeit, gritty paste.

A $20 \% \mathrm{v} / \mathrm{v}$ suspension of coarse particles in the carrier was used for the model suspensions in most of these tests. Note that if the model carrier fluid is replaced by the equivalent non-Newtonian slurry made from the fine and slime components then this would typically correspond to say a $75-80 \% \mathrm{w} / \mathrm{w}$ suspension of mine waste material in water.

\subsection{Inclined pipe tests}

Test were conducted at constant delivered solids concentration in the 100NB loop conveying the suspension at velocities corresponding to laminar flow of the carrier fluid, and transport characteristics are shown in Figure 5.

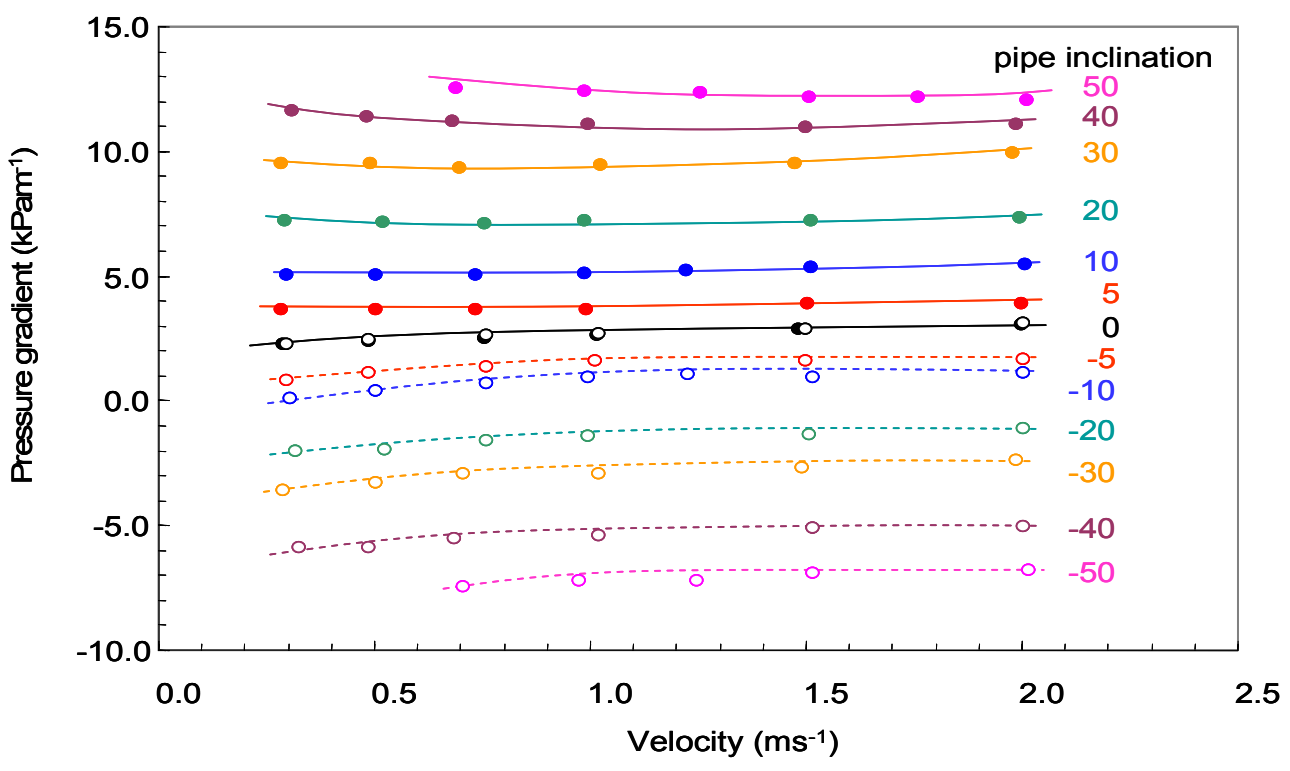

Figure 5 Transport characteristics for the suspension in pipes inclined from -50 to $50^{\circ}$ (solid symbols indicate flow going up, open symbols flow going down)

A sliding bed of coarse particles was observed for all flows, with the in-line solids concentration typically being higher for the up line than for the down line.

Coarse particle concentration maps obtained in the up and down legs of the loop are shown in Figure 6. 


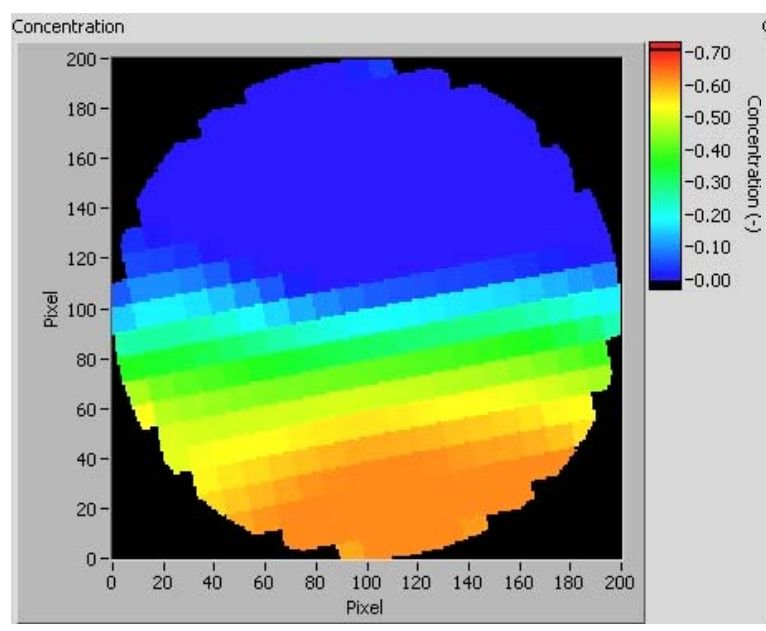

Up leg

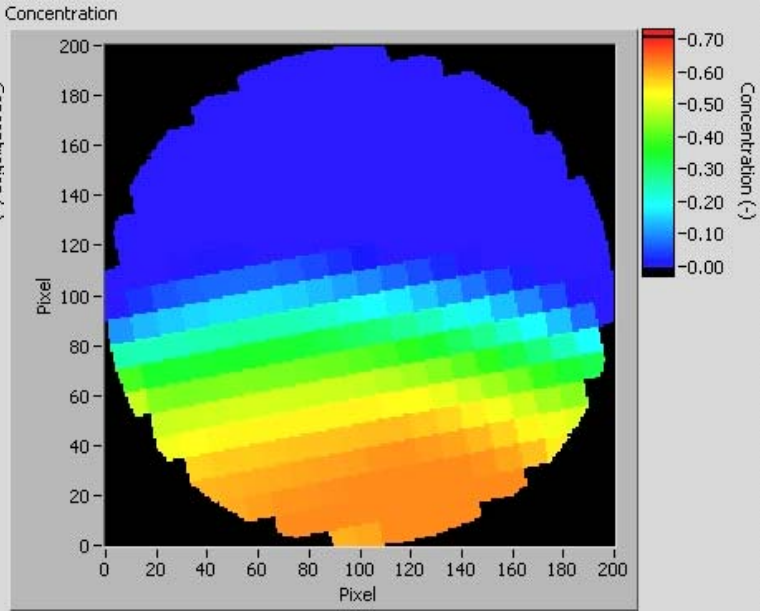

Down leg

\section{Figure 6 ERT concentration maps at $0.7 \mathrm{~ms}^{-1}$ and a pipe inclination of $5^{\circ}$}

\section{DISCUSSION}

The characteristic for the horizontal pipe, i.e $0^{\circ}$ inclination, in Figure 5, is similar to the rheogram shown in Figure 3 in shape although not value, i.e. they exhibit shear thinning characteristics, and this is a direct result of the non-Newtonian rheology of the carrier fluid. Characteristics for lines with a positive grade however become increasingly concave in shape, while those at negative grades become increasingly more convex. This is consistent with the sliding bed behaviour that was observed and is due to the changing in-line concentration required to maintain a constant delivered concentration under the various conditions.

If we assume, however, that the in-line concentration is reasonably constant with velocity, a reasonable assumption for high velocity flows, then it can be shown that for moderate pipe angles the relative change in pressure gradient with pipe inclination is simply

$$
\Delta p^{*}=\cos \theta+\frac{\sin \theta}{F \mu_{s}}
$$

where $\theta$ is the angle of inclination, $F$ a measure of the degree of fluidisation of the bed and $\mu_{s}$ the coefficient of sliding friction for the material (typically $0.3-0.5$ for mineral particles on steel). This equation assumes that there is no change in the fundamental flow structure in the pipe with inclination. Comparison between this equation and data taken from Figure 5 at $1.6 \mathrm{~ms}^{-1}$ is shown below. 


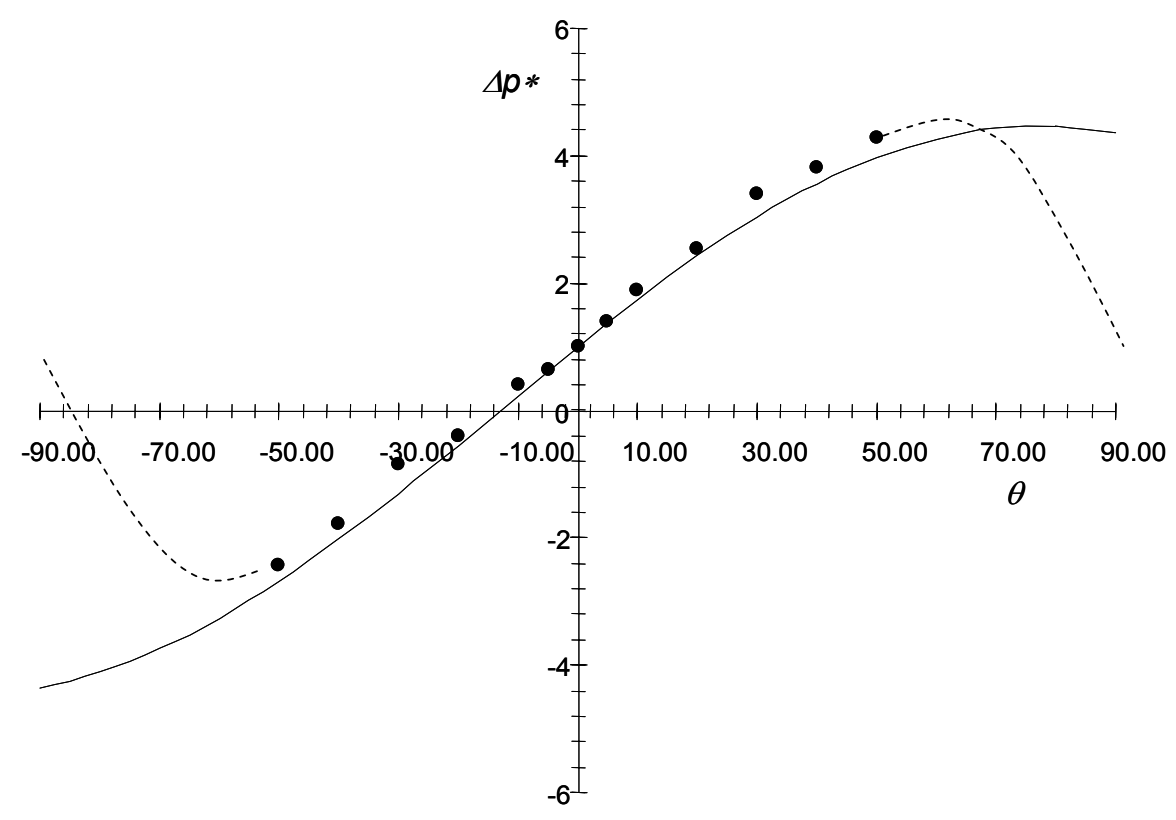

Figure $7 \quad$ Variation of relative pressure gradient with pipe inclination. Data shown for 1.6 $\mathrm{ms}^{-1}$

As shown, the agreement between with the data is quite good. Of surprise is that the agreement extends to $\pm 50^{\circ}$ as clearly the flow regime must change as the inclination steepens. Vertical flow, both up and down will be axisymetric, with a somewhat lower transport gradient than presented here and so the trend must change in a manner similar to that indicated by the dashed lines.

Investigations into the behaviour of Newtonian suspensions at various inclines have indicated that a limiting positive pipe angle is around $30^{\circ}$, corresponding to where the fluid velocity profile develops an inflection (Wilson and Tse 1984). Clearly the flow behaviour is different for these "paste" flows where the high viscosity ensures laminar flow and the coarse particles, i.e. $\mathrm{d}>20 \mu \mathrm{m}$, are conveyed as a sliding bed once the flow is fully established.

\section{CONCLUSIONS}

Preliminary data has been presented for a model co-disposal suspension pumped through a $100 \mathrm{NB}$ pipe at inclines varying from 0 to $\pm 50^{\circ}$. The flows, although statically stable and superficially appearing to be a homogeneous paste, stratified under shear to form a sliding bed over all flow rates and pipe inclinations tested. Prediction of the relative pressure gradient with pipe inclination, assuming that the mode of flow and in-line concentration were insensitive to pipe inclination, agreed well with the observed data.

The inclinable pipe test facility at Highett in Melbourne is fully commissioned and further investigation into these complex hybrid suspension flows, typical of mineral paste flows, will continue. 


\section{ACKNOWLEDGEMENTS}

The authors would like to thank the following sponsors of the AMIRA P599A project for funding this work and allowing this paper to be presented at Paste '06. CSIRO, BHP Billiton Ltd, Rio-Tinto Ltd, De Beers and the Weir Group.

\section{REFERENCES}

Erocolani, D., Ferrini, F. and Arrigoni, V. (1979) Electric and thermic probes for measuring the limit deposit velocity. Hydrotransport 6 : Int Conf on the Hydraulic Transport of Solids in Pipes, pp. 27-42.

Graham, L.J.W., Nguyen, B., Wu, J. and Kilpatrick, T. (2005) Improving wall velocity in non-Newtonian mixing tanks, 7th International Alumina Quality Workshop, Perth Australia, 16-21 October 2005.

Houman, J. (2003) Commissioning and operation of the paste thickening farm at Kimberley combined treatment plant. Pages Section 11 in 2003 International Seminar on Paste and Thickened Tailings. ACG, Melbourne.

Pullum, L. (2003) Pipeline Performance. in Paste 2003. Australian Centre for Geomechanics, Melbourne, Australia.

Pullum, L. and Graham, L.J.W. (2000) Paste pumping - true or false. in Paste 2000. ACG, Perth, Australia.

Pullum, L., Graham, L. and Slatter, P. (2004) A non-Newtonian two layer model and its application to high density hydrotransport. in 16th International Conference on Hydrotransport. BHR group, Santiago.

Talmon, A. and Mastbergen, D. (2004) Solids transport by drilling fluids: Concentrated bentonite-sand-slurries. pp. 641-649. in 12th Transport and Sedimentation of Solid Particles, Prague, Czech Republic.

Wilson, K.C., and Tse, J.K.P. (1984) Deposition limit for coarse-particle transport in inclined pipes. pp. 149-161. in Int. Conf. on Hydraulic Transport of Solids in Pipes. BHRA Fluid Engineering, Rome, Italy.

$\mathrm{Xu}$, J., Tipman, R., Gillies, R. and Shook, C. (2002) Centrifugal pump performance with Newtonian and non-Newtonian slurries. pp. 693-709. in Hydrotransport 15. BHR Group Limited, Banff, Canada. 